$$
\begin{aligned}
& \text { Algebra \& } \\
& \text { a } \\
& \begin{array}{l}
\text { Number } \\
\text { Theory }
\end{array} \\
& \text { Volume } 5
\end{aligned}
$$

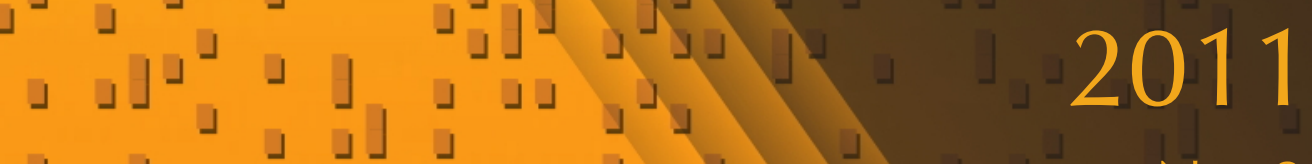

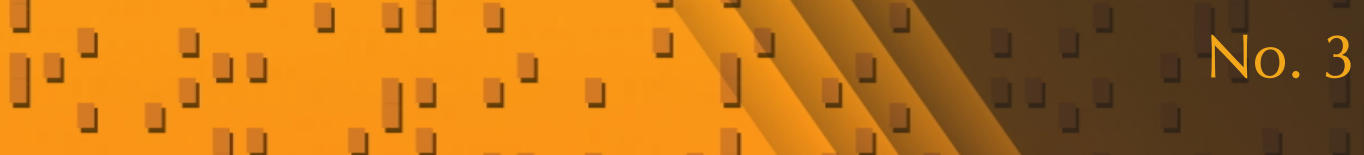

$$
\begin{aligned}
& \lrcorner
\end{aligned}
$$

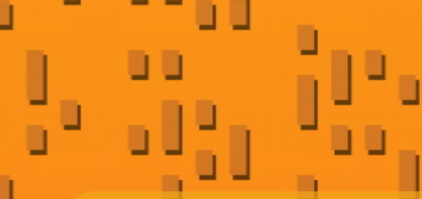

$$
\begin{aligned}
& \sqcup \sqcup \\
& \lrcorner\lrcorner\lrcorner\lrcorner\lrcorner
\end{aligned}
$$

Quantum differentiation and chain maps of bimodule complexes Anne V. Shepler and Sarah Witherspoon \lrcorner

」 


\title{
Quantum differentiation and chain maps of bimodule complexes
}

\author{
Anne V. Shepler and Sarah Witherspoon
}

\begin{abstract}
We consider a finite group acting on a vector space and the corresponding skew group algebra generated by the group and the symmetric algebra of the space. This skew group algebra illuminates the resulting orbifold and serves as a replacement for the ring of invariant polynomials, especially in the eyes of cohomology. One analyzes the Hochschild cohomology of the skew group algebra using isomorphisms which convert between resolutions. We present an explicit chain map from the bar resolution to the Koszul resolution of the symmetric algebra which induces various isomorphisms on Hochschild homology and cohomology, some of which have appeared in the literature before. This approach unifies previous results on homology and cohomology of both the symmetric algebra and skew group algebra. We determine induced combinatorial cochain maps which invoke quantum differentiation (expressed by Demazure-BGG operators).
\end{abstract}

\section{Introduction}

Let $G$ be a finite group acting linearly on a finite-dimensional complex vector space $V$. The skew group algebra $S(V) \# G$ is a natural semi-direct product of $G$ with the symmetric algebra $S(V)$ (a polynomial ring). It serves as a valuable, albeit noncommutative, replacement for the invariant ring $S(V)^{G}$ in geometric settings, as it encodes the abstract group structure of $G$ as well as its action on $V$. The cohomology of $S(V) \# G$ informs various areas of mathematics (for example, geometry, combinatorics, representation theory, and mathematical physics). In particular, the Hochschild cohomology of $S(V) \# G$ governs its deformations, which include graded Hecke algebras, symplectic reflection algebras, and Cherednik algebras.

\footnotetext{
Shepler was partially supported by NSF grants \#DMS-0402819 and \#DMS-0800951 and a research fellowship from the Alexander von Humboldt Foundation while in residence at RWTH Aachen University, Germany. Witherspoon was partially supported by NSA grant \#H98230-07-1-0038 and NSF grant \#DMS-0800832. Both authors were jointly supported by Advanced Research Program Grant 010366-0046-2007 from the Texas Higher Education Coordinating Board.
}

MSC2000: primary 16E40; secondary $16 \mathrm{~S} 35$.

Keywords: Hochschild cohomology, skew group algebra, Koszul resolution, Demazure-BGG operator, quantum differentiation. 
The orbifold $V / G$ may be realized as an algebraic variety whose coordinate ring is the ring of invariant functions $S\left(V^{*}\right)^{G}$ on the dual space $V^{*}$, which is the center of $S\left(V^{*}\right) \# G$ when $G$ acts faithfully. (For details, see [Harris 1995].) The variety $V / G$ is nonsingular exactly when the action of $G$ on $V$ is generated by reflections. Geometers and physicists are interested in resolving the singularities of $V / G$ with a smooth variety $X$ and examining the coordinate ring of $X$ instead of $S(V)^{G}$. In situations they study, the skew group algebra $S(V) \# G$ serves as a replacement for the coordinate ring of $X$; indeed, Hochschild cohomology sees no difference between these rings [Căldăraru et al. 2004]. Connections with representation theory are still unfolding; for example, see [Gordon and Smith 2004].

The Hochschild cohomology $\mathrm{HH}^{*}(A)$ of any algebra $A$ over a field $k$ is the space $\operatorname{Ext}_{A \otimes A^{o p}}(A, A)$. The cup product and Gerstenhaber bracket on Hochschild cohomology are both defined initially on the bar resolution, a natural $A \otimes A^{o p}$-free resolution of $A$. The cup product has another description as Yoneda composition of extensions of modules, which can be transported to any other projective resolution. However, the Gerstenhaber bracket has resisted such a general description. Instead, one commonly computes $\mathrm{HH}^{\circ}(A)$ using a more convenient resolution, then one finds and uses relevant chain maps to lift the Gerstenhaber bracket from the bar resolution. The case $A=S(V) \# G$ is complicated further because one does not work with resolutions of $A$ directly, but instead one derives information from resolutions of the symmetric algebra $S(V)$.

In this paper, we begin this task by constructing explicit chain maps which encode traffic between resolutions used to describe the Hochschild cohomology of $A=S(V) \# G$. Our maps convert between the bar and Koszul resolutions of the polynomial ring $S(V)$, and serve as a tool for investigating the homology and cohomology of $S(V)$ with coefficients in any bimodule. Specifically, the Koszul resolution of the polynomial ring $S(V)$ embeds naturally into the bar resolution. We define an explicit chain map, depending on a choice of basis, giving a quasi-inverse to this embedding. We study in particular the induced maps on the Hochschild cohomology $\mathrm{HH}^{*}(S(V), S(V) \# G)$. We give an elegant, combinatorial description of the induced map on cochains in terms of scaled Demazure (BGG) operators (or quantum partial differential operators, see Definition 3-4). We describe the induced maps on Hochschild homology as well. (These combinatorial descriptions are useful for computations, which we pursue in other articles.) The cohomology $\mathrm{HH}^{\circ}(S(V) \# G)$ manifests as the $G$-invariant subspace of $\mathrm{HH}^{\circ}(S(V), S(V) \# G)$ in characteristic 0 . We thus obtain isomorphisms of homology and cohomology that allow one to transfer structures defined on the bar resolution to the complexes standardly used to describe $\mathrm{HH}^{\circ}(S(V) \# G)$.

In Section 2, we establish notation and deploy the Hochschild cohomology $\mathrm{HH}^{\circ}(S(V) \# G)$ in terms of both the Koszul and bar resolutions of $S(V)$. We 
introduce a combinatorial map $\Upsilon$ on cochains in Section 3. This combinatorial converter $\Upsilon$ takes vector forms (tagged by group elements) to twisted quantum differential operators. In Section 4, we give a technical formula for explicit chain maps from the bar resolution to the Koszul resolution (Definition 4-1), which is valid over an arbitrary ground field. These specific chain maps each induce an inverse to the embedding of the Koszul resolution into the bar resolution after taking homology or cohomology. (Indeed, after applying functors $\otimes$ or Hom, we recover some chain maps given in the literature for converting between complexes expressing Hochschild homology and cohomology-see Section 6.) In Section 5, we deduce that our combinatorial converter $\Upsilon$ defines automorphisms of cohomology by showing that it is induced by the chain maps of Section 4 . We present similar automorphisms of homology (using quantum differentiation) in Section 6.

Our approach presents an immediate and obvious advantage: We define one primitive map between resolutions and then apply various functors that automatically give (co)chain maps in a variety of settings. We do not need to give separate proofs (depending on context) showing that these induced maps are chain maps, as such results follow immediately from the general theory. This uniform treatment provides a clear channel for navigating between chain and cochain complexes. Indeed, we use this channel in [Shepler and Witherspoon 2009; 2011] to explore the algebraic structure of $\mathrm{HH}^{\circ}(S(V) \# G)$ under the cup product and the Gerstenhaber bracket.

Some results in this paper are valid over a field of arbitrary characteristic, while others assume the ground field is the complex numbers, $\mathbb{C}$. We have tried to state carefully requirements on the field throughout. The reader should note that whenever we work over $\mathbb{C}$, we could instead work over any field containing the eigenvalues of the action of $G$ on $V$ in which $|G|$ is invertible. All tensor and exterior products will be taken over the ground field unless otherwise indicated.

\section{Preliminary material}

In this section, we work over the complex numbers $\mathbb{C}$, although the definitions below of Hochschild cohomology, bar resolution, and Koszul resolution are valid over any ground field.

Let $G$ be a finite group and $V$ a (not necessarily faithful) $\mathbb{C} G$-module. Let ${ }^{g} v$ denote the image of $v \in V$ under the action of $g \in G$. We work with the induced group action on all maps throughout this article: For any map $\theta$ and element $h \in$ GL $(V)$, we define the map ${ }^{h} \theta$ by $\left({ }^{h} \theta\right)(v):={ }^{h}\left(\theta\left({ }^{h^{-1}} v\right)\right)$ for all $v$. Let $V^{*}$ denote the vector space dual to $V$ with the contragredient (i.e., dual) representation. For any basis $v_{1}, \ldots, v_{n}$ of $V$, let $v_{1}^{*}, \ldots, v_{n}^{*}$ be the dual basis of $V^{*}$. Let $V^{G}=$ $\left\{v \in V:{ }^{g} v=v\right.$ for all $\left.g \in G\right\}$, the set of $G$-invariants in $V$. For any $g \in G$, let 
$Z(g)=\{h \in G: g h=h g\}$, the centralizer of $g$ in $G$, and let $V^{g}=\left\{v \in V:{ }^{g} v=v\right\}$, the $g$-invariant subspace of $V$.

The skew group algebra $S(V) \# G$ is the vector space $S(V) \otimes \mathbb{C} G$ with multiplication given by

$$
(a \otimes g)(b \otimes h)=a\left({ }^{g} b\right) \otimes g h
$$

for all $a, b \in S(V)$ and $g, h \in G$. We abbreviate $a \otimes g$ by $a \bar{g}(a \in S(V), g \in G)$ and $a \otimes 1,1 \otimes g$ simply by $a, \bar{g}$, respectively. An element $g \in G$ acts on $S(V)$ by an inner automorphism in $S(V) \# G: \bar{g} a(\bar{g})^{-1}=\left({ }^{g} a\right) \bar{g}(\bar{g})^{-1}={ }^{g} a$ for all $a \in A$.

The Hochschild cohomology of a $\mathbb{C}$-algebra $A$ (such as $A=S(V) \# G)$, with coefficients in an $A$-bimodule $M$, is the graded vector space

$$
\mathrm{HH}^{\bullet}(A, M)=\operatorname{Ext}_{A^{e}}(A, M),
$$

where $A^{e}=A \otimes A^{o p}$ acts on $A$ by left and right multiplication. This cohomology may be expressed in terms of the bar resolution, the following free $A^{e}$-resolution of $A$ :

$$
\cdots \stackrel{\delta_{3}}{\longrightarrow} A^{\otimes 4} \stackrel{\delta_{2}}{\longrightarrow} A^{\otimes 3} \stackrel{\delta_{1}}{\longrightarrow} A^{e} \stackrel{m}{\longrightarrow} A \rightarrow 0
$$

where

$$
\delta_{p}\left(a_{0} \otimes \cdots \otimes a_{p+1}\right)=\sum_{j=0}^{p}(-1)^{j} a_{0} \otimes \cdots \otimes a_{j} a_{j+1} \otimes \cdots \otimes a_{p+1},
$$

and $\delta_{0}=m$ is multiplication. We apply $\operatorname{Hom}_{A^{e}}(-, M)$ to obtain a cochain complex whose homology is $\mathrm{HH}^{\circ}(A, M)$. If $M=A$, we abbreviate $\mathrm{HH}^{\circ}(A)=\mathrm{HH}^{*}(A, A)$. For each $p$,

$$
\operatorname{Hom}_{A^{e}}\left(A^{\otimes(p+2)}, A\right) \cong \operatorname{Hom}_{\mathbb{C}}\left(A^{\otimes p}, A\right),
$$

and we identify these two spaces of $p$-cochains throughout this article. The graded vector space $\mathrm{HH}^{\circ}(A)$ admits both a cup product and a graded Lie bracket under which it becomes a Gerstenhaber algebra. In this article, we develop automorphisms of cohomology converting between resolutions. These automorphisms will be used in later publications to explore the algebraic structure of $\mathrm{HH}^{\circ}(S(V) \# G)$ under these two operations.

Hochschild cohomology of $\boldsymbol{S}(\boldsymbol{V}) \# \boldsymbol{G}$. Farinati [2005] and Ginzburg and Kaledin [2004] determined the graded vector space structure of $\mathrm{HH}^{\circ}(S(V) \# G)$ when $G$ acts faithfully on $V$. The same techniques apply to nonfaithful actions. The following statements are valid only when the characteristic does not divide the order of $G$. (Otherwise, the cohomology is more complicated as the group algebra of $G$ may itself not be semisimple.) Let $\mathscr{b}$ be a set of representatives of the conjugacy classes of $G$. A consequence of [Ştefan 1995, Corollary 3.4] posits a natural $G$-action 
giving the first of a series of isomorphisms of graded vector spaces:

$$
\begin{aligned}
\mathrm{HH}^{*}(S(V) \# G) & \cong \mathrm{HH}^{\cdot}(S(V), S(V) \# G)^{G} \\
& \cong\left(\bigoplus_{g \in G} \mathrm{HH}^{\cdot}(S(V), S(V) \bar{g})\right)^{G} \\
& \cong \bigoplus_{g \in \mathscr{C}} \mathrm{HH}^{*}(S(V), S(V) \bar{g})^{Z(g)} .
\end{aligned}
$$

Specifically, the action of $G$ on $V$ extends naturally to the bar complex of $S(V)$ and commutes with the differentials, and so induces a natural action on Hochschild cohomology $\mathrm{HH}^{*}(S(V), S(V) \# G)$ (for which we also use the action of $G$ on $S(V) \# G$ by inner automorphisms). The subspace of $G$-invariants of this action is denoted by $\mathrm{HH}^{*}(S(V), S(V) \# G)^{G}$. (Equivalently, this may also be defined via any other choice of $G$-compatible resolution used to compute cohomology; see [Ştefan 1995, Section 2], for example.)

The second isomorphism of (2-2) surfaces simply because the $S(V)^{e}$-module $S(V) \# G$ decomposes into the direct sum of $S(V)^{e}$-modules $S(V) \bar{g}$, and cohomology preserves direct sums. (The isomorphism arises of course at the cochain level, as the Hom-functor preserves direct sums.) We identify $\mathrm{HH}^{\circ}(S(V), S(V) \# G)$ with $\bigoplus_{g \in G} \mathrm{HH}^{*}(S(V), S(V) \bar{g})$ when convenient throughout this article. Note that $G$ permutes the components in the direct sum in accordance with the conjugation action of $G$ on itself. Thus for each $g \in G$, the subgroup $Z$ ( $g$ ) fixes the $g$-component $\mathrm{HH}^{*}(S(V), S(V) \bar{g})$ setwise. The third isomorphism of (2-2) canonically projects onto a set of representative summands.

One may use the Koszul resolution for $S(V)$ to determine each $g$-component $\mathrm{HH}^{\circ}(S(V), S(V) \bar{g})$ in the last line of (2-2) above. The Koszul resolution, denoted by $K_{.}(S(V))$, is given by $K_{0}(S(V))=S(V)^{e}, K_{1}(S(V))=S(V)^{e} \otimes V$, and for each $p \geq 2$,

$$
K_{p}(S(V))=\bigcap_{j=0}^{p-2} S(V)^{e} \otimes\left(V^{\otimes j} \otimes R \otimes V^{\otimes(p-j-2)}\right),
$$

where $R$ is the subspace of $V \otimes V$ spanned by all $v \otimes w-w \otimes v(v, w \in V)$; see [Braverman and Gaitsgory 1996], for example. This is a subcomplex of the bar resolution (2-1) for $S(V)$. For any choice of basis $v_{1}, \ldots, v_{n}$ of $V$, it is equivalent to the Koszul resolution corresponding to the regular sequence $\left\{v_{i} \otimes 1-1 \otimes v_{i}\right\}_{i=1}^{n}$ in $S(V)^{e}$ :

$$
K_{p}\left(\left\{v_{i} \otimes 1-1 \otimes v_{i}\right\}_{i=1}^{n}\right) \cong S(V)^{e} \otimes \bigwedge^{p}(V),
$$


a free $S(V)^{e}$-resolution of $S(V)$; see [Weibel 1994, §4.5], for instance. The differentials are given by

$d_{p}\left(1 \otimes 1 \otimes v_{j_{1}} \wedge \cdots \wedge v_{j_{p}}\right)$

$$
=\sum_{i=1}^{p}(-1)^{i+1}\left(v_{j_{i}} \otimes 1-1 \otimes v_{j_{i}}\right) \otimes\left(v_{j_{1}} \wedge \cdots \wedge \hat{v}_{j_{i}} \wedge \cdots \wedge v_{j_{p}}\right) .
$$

The canonical inclusion of the Koszul resolution (2-3) into the bar resolution (2-1) for $S(V)$ is then given on resolution (2-4) by the chain map

$$
\Phi: S(V)^{e} \otimes \wedge^{\bullet}(V) \rightarrow S(V)^{\otimes(\bullet+2)},
$$

defined by

$$
\Phi_{p}\left(1 \otimes 1 \otimes v_{j_{1}} \wedge \cdots \wedge v_{j_{p}}\right)=\sum_{\pi \in \operatorname{Sym}_{p}} \operatorname{sgn}(\pi) \otimes v_{j_{\pi(1)}} \otimes \cdots \otimes v_{j_{\pi(p)}} \otimes 1
$$

for all $v_{j_{1}}, \ldots, v_{j_{p}} \in V, p \geq 1$, where $\operatorname{Sym}_{p}$ denotes the symmetric group on $p$ symbols. Note that by its definition, $\Phi$ is invariant under the action of GL $(V)$, i.e., ${ }^{h} \Phi=\Phi$ for all $h$ in GL $(V)$.

Any chain map $\Psi_{p}: S(V)^{\otimes(p+2)} \rightarrow S(V)^{e} \otimes \bigwedge^{p} V$ from the bar resolution to the Koszul resolution yields a commutative diagram:

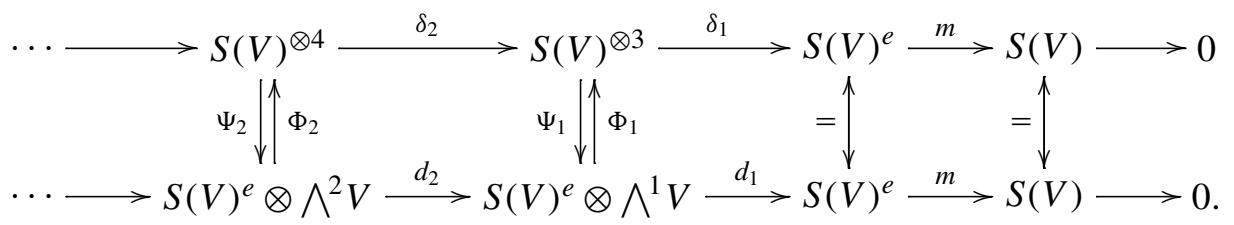

(In Definition 4-1, we explicitly define a map $\Psi$ depending on a choice of basis of $V$.) Such maps $\Phi$ and $\Psi$ necessarily induce inverse isomorphisms on cohomology $\mathrm{HH}^{*}(S(V), M)$ for any $S(V)$-bimodule $M$ upon applying $\operatorname{Hom}_{S(V)^{e}}(-, M)$. (Similarly for homology; see Section 6.) Identifying $\operatorname{Hom}_{S(V)^{e}}\left(S(V)^{\otimes(p+2)}, M\right)$ with $\operatorname{Hom}_{\mathbb{C}}\left(S(V)^{\otimes p}, M\right)$ and $\operatorname{Hom}_{S(V)^{e}}\left(S(V)^{e} \otimes \wedge^{p} V, M\right)$ with $\operatorname{Hom}_{\mathbb{C}}\left(\bigwedge^{p} V, M\right)$ for all $p$, we obtain the following commutative diagram:

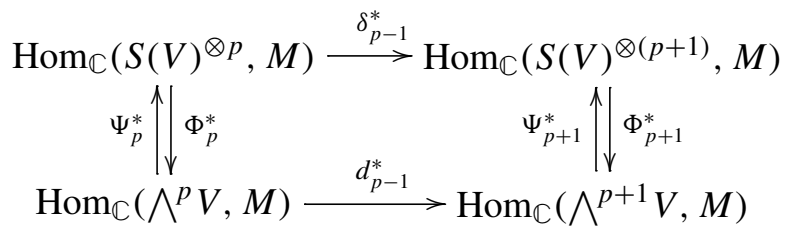

The maps $\Phi \Psi$ and $\Psi \Phi$ are each homotopic to an identity map by the Comparison Theorem, and thus $\Phi_{p}^{*}$ and $\Psi_{p}^{*}$ induce inverse automorphisms on the cohomology $\mathrm{HH}^{p}(S(V), M)$; see the proof of [Weibel 1994, Lemma 2.4.1]. In this paper, we primarily consider the $S(V)^{e}$-modules $M=S(V) \# G$ and $M=S(V) \bar{g}$ for $g$ in $G$. 
We transfer the map $\Phi$ and any chain map $\Psi$ to the Hochschild cohomology of the full skew group algebra, $\mathrm{HH}^{\circ}(S(V) \# G)$, using the isomorphisms of (2-2). Set $M=S(V) \# G$ and let $\Phi^{*}$ and $\Psi^{*}$ denote the induced maps on the cohomology

$$
\mathrm{HH}^{*}(S(V), S(V) \# G) \cong \bigoplus_{g \in G} \mathrm{HH}^{*}(S(V), S(V) \bar{g}) .
$$

For each $g$ in $G$, denote the restrictions to $\mathrm{HH}^{*}(S(V), S(V) \bar{g})$ by $\Phi_{g}^{*}$ and $\Psi_{g}^{*}$, respectively, so that

$$
\Phi^{*}=\bigoplus_{g \in G} \Phi_{g}^{*} \quad \text { and } \quad \Psi^{*}=\bigoplus_{g \in G} \Psi_{g}^{*} .
$$

The maps $\Phi^{*}$ and $\Psi^{*}$ behave nicely with respect to the action of $G$ :

Proposition 2-8. Let $\Psi$ be any choice of chain map from the bar resolution (2-1) to the Koszul resolution (2-4). The cochain maps $\Phi^{*}$ and $\Psi^{*}$ are inverse automorphisms on the cohomology $\mathrm{HH}^{*}(S(V), S(V) \# G)$ converting between expressions arising from the Koszul resolution and from the bar resolution. In addition,

(1) For any $g \in G$, the maps $\Phi_{g}^{*}$ and $\Psi_{g}^{*}$ on the cohomology $\mathrm{HH}^{*}(S(V), S(V) \bar{g})$ are invariant under the centralizer $Z(g)$ of $g$ in $G$, and the maps $\Phi^{*}$ and $\Psi^{*}$ on $\bigoplus_{g \in G} \mathrm{HH}^{*}(S(V), S(V) \bar{g})$ are invariant under $G$;

(2) The maps $\Phi^{*}$ and $\Psi^{*}$ induce inverse automorphisms on the graded vector space

$$
\bigoplus_{g \in \mathscr{C}}\left(\mathrm{HH}^{*}(S(V), S(V) \bar{g})\right)^{Z(g)} \cong\left(\bigoplus_{g \in G} \mathrm{HH}^{*}(S(V), S(V) \bar{g})\right)^{G} \cong \mathrm{HH}^{\cdot}(S(V) \# G) .
$$

Proof. As explained after Diagram (2-7), the maps $\Phi_{g}^{*}$ and $\Psi_{g}^{*}$ are inverse isomorphisms on the cohomology $\mathrm{HH}^{*}(S(V), S(V) \bar{g})$. By its definition, $\Phi$ is invariant under the action of $\mathrm{GL}(V)$, and so the map $\Phi^{*}$ on $\mathrm{HH}^{*}(S(V), S(V) \# G)$ is invariant under $G$, and the map $\Phi_{g}^{*}$ on $\mathrm{HH}^{\circ}(S(V), S(V) \bar{g})$ is invariant under $Z(g)$. Fix some $h$ in $Z(g)$ and consider the map ${ }^{h}\left(\Psi_{g}^{*}\right)$. As maps on the cohomology $\mathrm{HH}^{\bullet}(S(V), S(V) \bar{g})$,

$$
1={ }^{h}\left(\Phi_{g}^{*} \Psi_{g}^{*}\right)={ }^{h}\left(\Phi_{g}^{*}\right)^{h}\left(\Psi_{g}^{*}\right)=\Phi_{g}^{*} h\left(\Psi_{g}^{*}\right),
$$

thus ${ }^{h}\left(\Psi_{g}^{*}\right)$ is also inverse to $\Phi_{g}^{*}$. Hence ${ }^{h}\left(\Psi_{g}^{*}\right)=\Psi_{g}^{*}$ (since the inverse is unique) as maps on cohomology, for all $h$ in $Z(g)$, and $\Psi_{g}^{*}$ is also $Z(g)$-invariant. Thus statement (1) holds. As a consequence, we may restrict both $\Phi_{g}^{*}$ and $\Psi_{g}^{*}$ to the graded vector space $\left(\mathrm{HH}^{*}(S(V), S(V) \bar{g})\right)^{Z(g)}$. Applying the isomorphisms (2-2), we obtain (2).

The cohomology $\mathrm{HH}^{*}(S(V), S(V) \# G)$ arising from the Koszul resolution (2-4) of $S(V)$ may be viewed as a set of vector forms on $V$ tagged by group elements 
of $G$. Indeed, we identify $\operatorname{Hom}_{\mathbb{C}}\left(\bigwedge^{p} V, S(V) \bar{g}\right)$ with $S(V) \bar{g} \otimes \bigwedge^{p} V^{*}$ for each $g$ in $G$, and recognize the set of cochains derived from the Koszul resolution as (see Diagram (3-3) below)

$$
C^{\bullet}:=\bigoplus_{g \in G} C_{g}^{\cdot}, \quad \text { where } \quad C_{g}^{p}:=S(V) \bar{g} \otimes \bigwedge^{p} V^{*} .
$$

\section{Quantum differentiation and a combinatorial converter map}

One generally uses the Koszul resolution of $S(V)$ to compute Hochschild cohomology, but some of the algebraic structure of its cohomology is defined using the bar resolution instead. We thus define automorphisms of cohomology which convert between resolutions. In Equation (2-6), we defined the familiar inclusion map $\Phi$ from the Koszul resolution to the bar resolution. But in order to transfer algebraic structure, we need chain maps in both directions. In Section 4, we shall construct explicit chain maps $\Psi$ from the bar resolution to the Koszul resolution, which will then induce cochain maps $\Psi^{*}$. These maps $\Psi$ are somewhat unwieldy, however. Thus, in this section, we first define a more elegant and handy map $\Upsilon$ on cochains using quantum differential operators (alternatively, Demazure operators). In Theorem 5-1, we prove that $\Upsilon=\Psi^{*}$ as maps on cocycles, for our specific construction of a chain map $\Psi$ from the bar resolution to the Koszul resolution of $S(V)$. This implies that the map $\Upsilon$ is itself a cochain map, and that $\Upsilon$ is in fact equal to $\Psi^{*}$ on cohomology, for any choice of chain map $\Psi$ from the bar resolution to the Koszul resolution of $S(V)$. This development allows us to deduce important properties of the expedient map $\Upsilon$ (useful for computations) from the elephantine map $\Psi^{*}$. In this section, we work over the complex numbers $\mathbb{C}$.

Given any basis $v_{1}, \ldots, v_{n}$ of $V$, and any complex number $\epsilon \neq 1$, we define the $\epsilon$-quantum partial differential operator with respect to $v:=v_{i}$ as the scaled Demazure (BGG) operator $\partial_{v, \epsilon}: S(V) \rightarrow S(V)$ given by

$$
\partial_{v, \epsilon}(f)=(1-\epsilon)^{-1} \frac{f-{ }^{s} f}{v}=\frac{f-{ }^{s} f}{v-{ }^{s} v},
$$

where $s \in \mathrm{GL}(V)$ is the reflection whose matrix with respect to the basis $v_{1}, \ldots, v_{n}$ is $\operatorname{diag}(1, \ldots, 1, \epsilon, 1, \ldots, 1)$ with $\epsilon$ in the $i$ th slot. Set $\partial_{v, \epsilon}=\partial / \partial v$, the usual partial differential operator with respect to $v$, when $\epsilon=1$.

Remark 3-2. The quantum partial differential operator $\partial_{v, \epsilon}$ above coincides with the usual definition of quantum partial differentiation: One takes the ordinary partial derivative with respect to $v$ but instead of multiplying each monomial by its degree $k$ in $v$, one multiplies by the quantum integer $[k]_{\epsilon}$, where

$$
[k]_{\epsilon}:=1+\epsilon+\epsilon^{2}+\cdots+\epsilon^{k-1} .
$$


Let us check explicitly that these two definitions coincide. For $v=v_{1}, \epsilon \neq 1$,

$$
\begin{aligned}
& \partial_{v, \epsilon}\left(v_{1}^{k_{1}} v_{2}^{k_{2}} \cdots v_{n}^{k_{n}}\right)=\frac{\left(v_{1}^{k_{1}} v_{2}^{k_{2}} \cdots v_{n}^{k_{n}}\right)-{ }^{s}\left(v_{1}^{k_{1}} v_{2}^{k_{2}} \cdots v_{n}^{k_{n}}\right)}{v_{1}-{ }^{s} v_{1}} \\
& =\frac{v_{1}^{k_{1}} v_{2}^{k_{2}} \cdots v_{n}^{k_{n}}-\epsilon^{k_{1}} v_{1}^{k_{1}} v_{2}^{k_{2}} \cdots v_{n}^{k_{n}}}{v_{1}-\epsilon v_{1}} \\
& =\frac{\left(1-\epsilon^{k_{1}}\right) v_{1}^{k_{1}} v_{2}^{k_{2}} \cdots v_{n}^{k_{n}}}{(1-\epsilon) v_{1}} \\
& =\left[k_{1}\right]_{\epsilon} v_{1}^{k_{1}-1} v_{2}^{k_{2}} \cdots v_{n}^{k_{n}} .
\end{aligned}
$$

We are now ready to construct the map $\Upsilon$ taking vector forms (tagged by group elements) to twisted quantum differential operators. We define $\Upsilon$ on cochains $C^{\bullet}$ (see (2-9)) so that the following diagram commutes for $M=S(V) \# G$ :

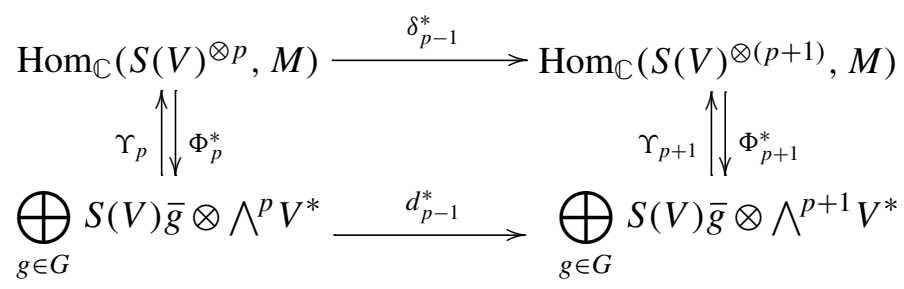

First, some notation. For $g$ in $G$, fix a basis $B_{g}=\left\{v_{1}, \ldots, v_{n}\right\}$ of $V$ consisting of eigenvectors of $g$ with corresponding eigenvalues $\epsilon_{1}, \ldots, \epsilon_{n}$. Decompose $g$ into a product of reflections diagonal in this basis: Let $g=s_{1} \cdots s_{n}$ where each $s_{i}$ is either the identity or a reflection defined by ${ }^{s_{i}} v_{j}=v_{j}$ for $j \neq i$ and ${ }^{s_{i}} v_{i}=\epsilon_{i} v_{i}$. Let $\partial_{i}:=\partial_{v_{i}, \epsilon_{i}}$, the quantum partial derivative (see Definition (3-1)) with respect to $B_{g}$.

Definition 3-4. We define a resolution converter map $\Upsilon$ from the dual Koszul complex to the dual bar complex with coefficients in $S(V) \# G$ :

$$
\Upsilon_{p}: C^{p} \rightarrow \operatorname{Hom}_{\mathbb{C}}\left(S(V)^{\otimes p}, S(V) \# G\right) .
$$

Let $g$ lie in $G$ with basis $B_{g}=\left\{v_{1}, \ldots, v_{n}\right\}$ of $V$ as above. Let

$$
\alpha=f_{g} \bar{g} \otimes v_{j_{1}}^{*} \wedge \cdots \wedge v_{j_{p}}^{*}
$$

with $f_{g} \in S(V)$ and $1 \leq j_{1}<\ldots<j_{p} \leq n$. Define $\Upsilon(\alpha): S(V)^{\otimes p} \rightarrow S(V) \# G$ by

$$
\Upsilon(\alpha)\left(f_{1} \otimes \cdots \otimes f_{p}\right)=\left(\prod_{k=1}^{p} s_{1} s_{2} \cdots s_{j_{k}-1}\left(\partial_{j_{k}} f_{k}\right)\right) f_{g} \bar{g} .
$$

By Theorem 5-1 below, $\Upsilon$ is a cochain map. Thus $\Upsilon$ induces a map on the cohomology $\mathrm{HH}^{*}(S(V), S(V) \# G) \cong \bigoplus_{g \in G} \mathrm{HH}^{*}(S(V), S(V) \bar{g})$, which we denote by $\Upsilon$ 
as well. For each $g$ in $G$, let $\Upsilon_{g}$ denote the restriction to $C_{g}^{*}$ and the restriction to $\mathrm{HH}^{*}(S(V), S(V) \bar{g})$, so that

$$
\Upsilon=\bigoplus_{g \in G} \Upsilon_{g}
$$

Remark 3-5. For each $g$ in $G$, the cochain map $\Upsilon_{g}=\Upsilon_{g, B}$ depends on the chosen basis $B=B_{g}$ of eigenvectors of $g$. But we shall see (in Corollary 5-3 below) that the induced automorphism on cohomology $\mathrm{HH}^{\circ}(S(V), S(V) \bar{g})$ does not depend on the choice of basis. This will imply that as an automorphism of $\mathrm{HH}^{\circ}(S(V) \# G)$, the map $\Upsilon$ does not depend on choices of bases of $V$ used in its definition.

Example 3-6. Let $G=\mathbb{Z} / 2 \mathbb{Z} \times \mathbb{Z} / 2 \mathbb{Z}$ be the Klein four-group consisting of elements $1, g, h, g h$. Let $V=\mathbb{C}^{3}$ with basis $v_{1}, v_{2}, v_{3}$ on which $G$ acts by ${ }^{g} v_{1}=-v_{1}$, ${ }^{g} v_{2}=v_{2},{ }^{g} v_{3}=-v_{3},{ }^{h} v_{1}=-v_{1},{ }^{h} v_{2}=-v_{2},{ }^{h} v_{3}=v_{3}$. Let $\alpha=f_{h} \bar{h} \otimes v_{1}^{*} \wedge v_{2}^{*}$ for some $f_{h} \in S(V)$. Write $h=s_{1} s_{2}$, a product of reflections with ${ }^{s_{1}} v_{1}=-v_{1}$, ${ }^{s_{2}} v_{2}=-v_{2}$. Then $\Upsilon(\alpha)$ is the function on $S(V)^{\otimes 2}$ given by

$$
\Upsilon(\alpha)\left(f_{1} \otimes f_{2}\right)=\left(\partial_{1} f_{1}\right)\left({ }^{s_{1}} \partial_{2} f_{2}\right) f_{h} \bar{h}
$$

for all $f_{1}, f_{2} \in S(V)$. For example, $\Upsilon(\alpha)\left(v_{1} \otimes v_{2}\right)=f_{h} \bar{h}$ while $\Upsilon(\alpha)\left(v_{2} \otimes v_{1}\right)=0$.

Remark 3-7. The map $\Upsilon$ transforms any decomposable vector form into a (twisted) quantum operator characterizing the same subspace: For the fixed basis $B_{g}=$ $\left\{v_{1}, \ldots, v_{n}\right\}$ and $\alpha=f_{g} \bar{g} \otimes v_{j_{1}}^{*} \wedge \cdots \wedge v_{j_{p}}^{*}$ in $C_{g}^{p}$ (with $j_{1}<\ldots<j_{p}$ ), we have

$$
\Upsilon(\alpha)\left(v_{i_{1}} \otimes \cdots \otimes v_{i_{p}}\right)=0 \quad \text { unless } i_{1}=j_{1}, \ldots, i_{p}=j_{p} .
$$

Generally, $\Upsilon(\alpha)\left(f_{1} \otimes \cdots \otimes f_{p}\right)=0$ whenever $\frac{\partial}{\partial v_{j_{k}}}\left(f_{k}\right)=0$ for some $k$.

The next proposition explains how $\Upsilon$ depends on our choices of bases as a map on cochains.

Proposition 3-8. The maps $\Upsilon_{g, B}$ on cochains, for $g$ in $G$, satisfy the following change of basis rule: For any $a$ in $G$,

$$
{ }^{a} \Upsilon_{g, B}=\Upsilon_{a g a^{-1},{ }_{B}{ }_{B}} .
$$

In particular, for $a$ in the centralizer $Z(g),{ }^{a} \Upsilon_{g, B}=\Upsilon_{g,{ }^{a} B}$.

Proof. One may check directly from (3-1) that quantum partial differentiation obeys the following transformation law: For all $v$ in $V$ and $\epsilon$ in $\mathbb{C}$,

$$
{ }^{a} \partial_{v, \epsilon}=\partial a_{v, \epsilon},
$$

where ${ }^{a} \partial_{v, \epsilon}$ differentiates with respect to a basis $B$ and $\partial a_{v, \epsilon}$ with respect to ${ }^{a} B$.

Let $B=\left\{v_{1}, \ldots, v_{n}\right\}$ be a basis of $V$ of eigenvectors of $g$ with corresponding eigenvalues $\epsilon_{1}, \ldots, \epsilon_{n}$. Decompose $g$ as a product of diagonal reflections $s_{i}$ in $\mathrm{GL}(V)$ (for $i=1, \ldots, n$ ) in this basis; we retain the notation before Definition 3-4. 
Let $g^{\prime}=a g a^{-1}, B^{\prime}={ }^{a} B, v_{i}^{\prime}={ }^{a} v_{i}, s_{i}^{\prime}=a s_{i} a^{-1}$, and $\partial_{i}^{\prime}={ }^{a} \partial_{i}$. Then the $s_{i}^{\prime}$ similarly decompose $g^{\prime}$ in the basis $B^{\prime}$ with $\partial_{i}^{\prime}=\partial_{v_{i}^{\prime}, \epsilon_{i}}$.

Consider $\alpha=f_{g} \otimes v_{j_{1}}^{*} \wedge \cdots \wedge v_{j_{p}}^{*}$ in $C^{p}$. For all $f_{i}$ in $S(V)$,

$$
\begin{aligned}
{ }^{a}\left(\Upsilon_{g, B}(\alpha)\right)\left(f_{1} \otimes \ldots \otimes f_{p}\right) & ={ }^{a}\left(\Upsilon_{g, B}(\alpha)\left({ }^{a^{-1}} f_{1} \otimes \cdots \otimes{ }^{a^{-1}} f_{p}\right)\right) \\
& ={ }^{a}\left({ }^{s_{1} \cdots s_{j_{1}-1}} \partial_{j_{1}}\left({ }^{a^{-1}} f_{1}\right) \ldots{ }^{s_{1} \cdots s_{j_{p}-1}}\left(\partial_{j_{p}}\left({ }^{a^{-1}} f_{p}\right)\right) f_{g} \bar{g}\right) \\
& ={ }^{s_{1}^{\prime} \cdots s_{j_{1}-1}^{\prime}}\left({ }^{a} \partial_{j_{1}} f_{1}\right) \ldots s_{1}^{\prime} \cdots s_{j_{p}-1}^{\prime}\left({ }^{a} \partial_{j_{p}} f_{p}\right){ }^{a} f_{g} \bar{g}^{\prime} \\
& ={ }^{s_{1}^{\prime} \cdots s_{j_{1}-1}^{\prime}}\left(\partial_{j_{1}}^{\prime} f_{1}\right) \ldots s_{1}^{\prime} \cdots s_{j_{p}-1}^{\prime}\left(\partial_{j_{p}}^{\prime} f_{p}\right){ }^{a} f_{g} \bar{g}^{\prime} \\
& =\Upsilon_{g^{\prime}, B^{\prime}}\left({ }^{a} \alpha\right)\left(f_{1} \otimes \ldots \otimes f_{p}\right),
\end{aligned}
$$

and the result follows.

The above proposition can also be seen using Definition 4-1 below of the chain map $\Psi_{B}$, Theorem 5-1 below equating $\Upsilon_{g, B}$ and $\Psi_{B}$, and the straightforward fact that $\Psi_{B}$ has a similar change of basis property.

\section{Chain maps from the bar to the Koszul resolution}

In this section, we define specific chain maps $\Psi_{B}$ from the bar resolution of $S(V)$ to its Koszul resolution (see (2-1) and (2-4)) depending on bases $B$ of $V$. By the Comparison Theorem, the resulting maps $\left(\Psi_{B}\right)^{*}$ on cohomology do not depend on the choice of $B$. In particular, we consider cohomology with coefficients in $S(V) \bar{g}$ and write $\Psi_{g, B}^{*}$ for the induced map $\left(\Psi_{B}\right)_{g}^{*}$ on $\mathrm{HH}^{\circ}(S(V), S(V) \bar{g})$. We shall show in Theorem 5-1 below that $\Psi_{g, B}^{*}=\Upsilon_{g, B}$ (recall Definition 3-4) for any choice $B$ of basis of $V$ consisting of eigenvectors of $g$ used to define both maps. This will imply (see Corollary 5-3) that as maps on cohomology, $\Upsilon_{g}$ and $\Upsilon$ are automorphisms independent of choices of bases $B$ used to define them at the cochain level. In this section, we work over any base field.

First, we introduce some notation. Let $\underline{\ell}$ denote an $n$-tuple $\underline{\ell}:=\left(\ell_{1}, \ldots, \ell_{n}\right)$. Let $\underline{v}^{\underline{\ell}}$ be the monomial $\underline{v}^{\underline{\ell}}:=v_{1}^{\ell_{1}} \cdots v_{n}^{\ell_{n}}$ where $v_{1}, \ldots, v_{n}$ is a chosen basis of $V$. Sometimes we further abbreviate a $p$-tuple $\underline{\ell}^{1}, \ldots, \underline{\ell}^{p}$ of $n$-tuples by $\underline{\ell}$ when no confusion will arise.

Definition 4-1. Let $V$ be a vector space over an arbitrary field, and let $B=$ $\left\{v_{1}, \ldots, v_{n}\right\}$ be a basis of $V$. Define an $S(V)^{e}$-map $\Psi_{B}$ from the bar resolution to the Koszul resolution, $\Psi_{B}: S(V)^{\otimes(\cdot+2)} \rightarrow S(V)^{e} \otimes \wedge^{\bullet}(V)$, as follows. Let $\left(\Psi_{B}\right)_{0}$ be the identity map. For each $p \geq 1$, define $\left(\Psi_{B}\right)_{p}$ by

$$
\begin{aligned}
\left(\Psi_{B}\right)_{p} & \left(1 \otimes \underline{v}^{\underline{\ell^{1}}} \otimes \cdots \otimes \underline{v}^{\underline{\ell^{p}}} \otimes 1\right) \\
& =\sum_{1 \leq i_{1}<\cdots<i_{p} \leq n} \sum_{0 \leq a_{i_{j}}<\ell_{i_{j}}^{j}} \underline{\underline{v}} \underline{\underline{\ell}} \underline{\left.\underline{\ell} ; i_{1}, \ldots, i_{p}\right)} \otimes \underline{v} \underline{\underline{Q}} \underline{\left(\underline{\ell} ; i_{1}, \ldots, i_{p}\right)} \otimes v_{i_{1}} \wedge \cdots \wedge v_{i_{p}},
\end{aligned}
$$


where the second sum ranges over all $a_{i_{1}}, \ldots, a_{i_{p}}$ such that $0 \leq a_{i_{j}}<\ell_{i_{j}}^{j}$ for each $j \in\{1, \ldots, p\}$ and the functions $\underline{Q}$ and $\underline{\hat{Q}}$ (indicating monomial degree) depend also on the choices $a_{i_{j}}$ (this dependence is suppressed in the notation for brevity):

$$
\underline{Q}\left(\underline{\ell}^{1}, \ldots, \underline{\ell}^{p} ; i_{1}, \ldots, i_{p}\right)_{i}= \begin{cases}a_{i}+\ell_{i}^{1}+\cdots+\ell_{i}^{j-1} & \text { if } i=i_{j}, \\ \ell_{i}^{1}+\cdots+\ell_{i}^{j} & \text { if } i_{j}<i<i_{j+1},\end{cases}
$$

where we set $i_{0}=0$ and $i_{p+1}=n+1$ for convenience. We define the $n$-tuple $\underline{\hat{Q}}\left(\underline{\ell} ; i_{1}, \ldots, i_{p}\right)$ to be complementary to $\underline{Q}\left(\underline{\ell} ; i_{1}, \ldots, i_{p}\right)$ in the sense that

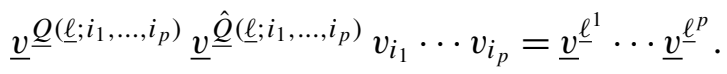

We simply write $\underline{\hat{Q}}$ when it is clear with respect to which $\underline{Q}\left(\underline{\ell} ; i_{1}, \ldots, i_{p}\right)$ it is complementary.

For small values of $p$, the formula for $\left(\Psi_{B}\right)_{p}$ is less cumbersome. In particular, for $p=1,2$, such formulas were given in [Witherspoon 2006, (4.9), (4.10)]. We repeat them here:

$$
\begin{aligned}
& \Psi_{1}\left(1 \otimes v_{1}^{\ell_{1}} \cdots v_{n}^{\ell_{n}} \otimes 1\right)=\sum_{i=1}^{n} \sum_{t=1}^{\ell_{i}} v_{i}^{\ell_{i}-t} v_{i+1}^{\ell_{i+1}} \cdots v_{n}^{\ell_{n}} \otimes v_{1}^{\ell_{1}} \cdots v_{i-1}^{\ell_{i-1}} v_{i}^{t-1} \otimes v_{i}, \\
& \Psi_{2}\left(1 \otimes v_{1}^{\ell_{1}} \cdots v_{n}^{\ell_{n}} \otimes v_{1}^{m_{1}} \cdots v_{n}^{m_{n}} \otimes 1\right) \\
& =\sum_{1 \leq i<j \leq n} \sum_{r=1}^{m_{j}} \sum_{t=1}^{\ell_{i}}\left(v_{i}^{\ell_{i}-t} v_{i+1}^{\ell_{i+1}} \cdots v_{j-1}^{\ell_{j-1}} v_{j}^{\ell_{j}+m_{j}-r} v_{j+1}^{\ell_{j+1}+m_{j+1}} \cdots v_{n}^{\ell_{n}+m_{n}}\right. \\
& \left.\otimes v_{1}^{\ell_{1}+m_{1}} \cdots v_{i-1}^{\ell_{i-1}+m_{i-1}} v_{i}^{m_{i}+t-1} v_{i+1}^{m_{i+1}} \cdots v_{j-1}^{m_{j-1}} v_{j}^{r-1} \otimes v_{i} \wedge v_{j}\right) .
\end{aligned}
$$

Example 4-3. To illustrate, we compute $\Psi_{2}$ on a few monomials of small degree:

$\Psi_{2}\left(1 \otimes v_{1} \otimes v_{2} \otimes 1\right)=1 \otimes 1 \otimes v_{1} \wedge v_{2}$,

$$
\Psi_{2}\left(1 \otimes v_{1} v_{2} \otimes v_{2}^{3} \otimes 1\right)=\left(v_{2}^{3} \otimes 1+v_{2}^{2} \otimes v_{2}+v_{2} \otimes v_{2}^{2}\right) \otimes v_{1} \wedge v_{2},
$$

$\Psi_{2}\left(1 \otimes v_{1} v_{2} \otimes v_{2}^{2} v_{3} \otimes 1\right)=\left(v_{2}^{2} v_{3} \otimes 1+v_{2} v_{3} \otimes v_{2}\right) \otimes v_{1} \wedge v_{2}$

$$
+1 \otimes v_{1} v_{2}^{2} \otimes v_{2} \wedge v_{3}+v_{2} \otimes v_{2}^{2} \otimes v_{1} \wedge v_{3} .
$$

Theorem 4-4. For each choice of basis $B$ of $V$, the map $\Psi_{B}$ of Definition 4-1 is a chain map.

We defer the proof of Theorem 4-4 to the Appendix as it is rather technical.

\section{Merits of the combinatorial converter map}

In the previous two sections, we examined two maps $\Upsilon_{g, B}$ and $\Psi_{g, B}^{*}$ which convert between cochain complexes: They each transform cochains procured from 
the Koszul resolution (2-4) of $S(V)$ into cochains procured from the bar resolution (2-1) of $S(V)$ (see Definitions 3-4 and 4-1). In this section, we show that the two maps $\Upsilon_{g, B}$ and $\Psi_{g, B}^{*}$ are identical on cochains, and hence also on cohomology, for any $g$ in $G$ and any basis $B$ consisting of eigenvectors of $g$. This will imply that $\Upsilon$ is itself a cochain map. We deduce other salient properties of the map $\Upsilon$ using this connection between $\Upsilon$ and $\Psi$. We take our ground field to be $\mathbb{C}$ in this section.

Theorem 5-1. Let $g$ be in $G$ and let $B$ be a basis of $V$ consisting of eigenvectors of $g$. Then

$$
\Upsilon_{g, B}=\Psi_{g, B}^{*}
$$

as maps on cochains. Thus $\Upsilon_{g, B}$ is a cochain map.

Proof. We check that $\Upsilon_{g, B}$ and $\Psi_{g, B}^{*}$ agree on cochains: Let $\alpha=f_{g} \bar{g} \otimes v_{j_{1}}^{*} \wedge \cdots \wedge v_{j_{p}}^{*}$ be a cochain in $C_{g}^{p}$ with $f_{g} \in S(V)$ and $j_{1}<\ldots<j_{p}$, where $B=\left\{v_{1}, \ldots, v_{n}\right\}$. Let

$$
f_{1}=\underline{v}^{\underline{\ell}} \underline{\underline{\ell}}^{1}, \quad \ldots, \quad f_{p}=\underline{v}^{\underline{\ell}^{p}}
$$

be monomials in $S(V)$. Without loss of generality, it suffices to show that $\Psi_{g, B}^{*}(\alpha)$ and $\Upsilon_{g, B}(\alpha)$ agree on $f_{1} \otimes \cdots \otimes f_{p}$, since such elements form a basis for $S(V)^{\otimes p}$. By Definition 4-1,

$$
\begin{aligned}
\Psi_{g, B}^{*}(\alpha) & \left(f_{1} \otimes \cdots \otimes f_{p}\right) \\
= & \alpha\left(\Psi_{g, B}\left(f_{1} \otimes \cdots \otimes f_{p}\right)\right) \\
= & \alpha\left(\Psi_{g, B}\left(v_{1}^{\ell_{1}^{1}} \cdots v_{n}^{\ell_{n}^{1}} \otimes \cdots \otimes v_{1}^{\ell_{1}^{p}} \cdots v_{n}^{\ell_{n}^{p}}\right)\right) \\
& =\alpha\left(\sum_{1 \leq i_{1}<\cdots<i_{p} \leq n} \sum_{0 \leq a_{i_{k}}<\ell_{i_{k}}^{k}} \underline{v} \underline{\underline{Q}\left(\ell ; i_{1}, \ldots, i_{p}\right)} \otimes \underline{v} \underline{\underline{Q}} \underline{\left(\ell ; i_{1}, \ldots, i_{p}\right)} \otimes v_{i_{1}} \wedge \cdots \wedge v_{i_{p}}\right) .
\end{aligned}
$$

Since $\alpha$ has exterior part $v_{j_{1}}^{*} \wedge \cdots \wedge v_{j_{p}}^{*}$, each summand is zero save one (the summand with $i_{k}=j_{k}$ for $k=1, \ldots p$ ). Then

$$
\begin{aligned}
& \Psi_{g, B}^{*}(\alpha)\left(f_{1} \otimes \cdots \otimes f_{p}\right) \\
& =\sum_{0 \leq a_{j_{k}}<\ell_{j_{k}}^{k}} \underline{v} \underline{Q} \underline{\left(\ell ; j_{1}, \ldots, j_{p}\right)} f_{g} \bar{g} \underline{v} \underline{\hat{Q}} \underline{\ell}\left(j_{1}, \ldots, j_{p}\right) \\
& =\sum_{0 \leq a_{j_{k}}<\ell_{j_{k}}^{k}}\left(\prod_{t=1}^{p} \epsilon_{j_{t}}^{\left(\ell_{j t}^{t}-a_{j_{t}}-1\right)+\ell_{j t}^{t+1} \ldots+\ell_{j_{t}}^{p}} \prod_{i_{t-1}<i<i_{t}} \epsilon_{i}^{\ell_{i}^{t}+\cdots+\ell_{i}^{p}}\right) \underline{v} \underline{\underline{Q}} \underline{\left(\ell ; j_{1}, \ldots, j_{p}\right)} \underline{\underline{v}} \underline{\underline{Q}} \underline{\left(\ell ; j_{1}, \ldots, j_{p}\right)} f_{g} \bar{g} .
\end{aligned}
$$

Recall that, by definition, $\underline{v} \underline{Q}^{\left(\ell ; j_{1}, \ldots, j_{p}\right)} \underline{\underline{v}} \underline{\hat{Q}\left(\underline{\ell} ; j_{1}, \ldots, j_{p}\right)} v_{j_{1}} \cdots v_{j_{p}}=\underline{v}^{\underline{\ell}} \cdots \underline{\underline{\ell}}^{1}$. Thus the factor $\underline{v} \underline{Q} \underline{Q}\left(\underline{\ell} ; j_{1}, \ldots, j_{p}\right) \underline{v} \underline{\hat{Q}} \underline{\hat{\ell}} \underline{\left.\underline{j_{1}}, \ldots, j_{p}\right)} f_{g} \bar{g}$ in each term of the above sum does not depend on the values of $a_{j_{k}}$, and we may move the summation symbol inside the 
parentheses. Simplifying, we obtain

$$
\begin{aligned}
& \left(\prod_{t=1}^{p}\left[\ell_{j_{t}}^{t}\right]_{j_{t}} \epsilon_{j_{t}}^{\ell_{j t}^{t+1}+\cdots+\ell_{j_{t}}^{p}} \prod_{i_{t-1}<i<i_{t}} \epsilon_{i}^{\ell_{i}^{t}+\cdots+\ell_{i}^{p}}\right) \underline{v} \underline{\underline{Q}} \underline{\left(\ell, j_{1}, \ldots, j_{p}\right)} \underline{\underline{v}} \underline{\hat{Q}\left(\underline{\ell} ; j_{1}, \ldots, j_{p}\right)} f_{g} \bar{g} \\
& =\left(\prod_{k=1}^{p} s_{1} \cdots s_{j_{k}-1}\left(\partial_{j_{k}} f_{k}\right)\right) f_{g} \bar{g} \\
& =\Upsilon_{g, B}(\alpha)\left(f_{1} \otimes \cdots \otimes f_{p}\right),
\end{aligned}
$$

by Definition 3-4. Hence $\Upsilon_{g, B}=\Psi_{g, B}^{*}$ as maps on cochains.

As a consequence, we obtain a statement about any chain map $\Psi$ from the bar resolution to the Koszul resolution, at the level of cohomology, which has further implications for the map $\Upsilon$ :

Corollary 5-2. Let $\Psi$ be any chain map from the bar resolution (2-1) to the Koszul resolution (2-4) for $S(V)$. Then:

(1) $\Upsilon_{g}=\Psi_{g}^{*}$ as maps on the cohomology $\mathrm{HH}^{*}(S(V), S(V) \bar{g})$, for all $g$ in $G$.

(2) $\Upsilon=\Psi^{*}$ as maps on

$$
\mathrm{HH}^{*}(S(V), S(V) \# G) \cong \bigoplus_{g \in G} \mathrm{HH}^{*}(S(V), S(V) \bar{g})
$$

and on its $G$-invariant subalgebra,

$$
\mathrm{HH}^{*}(S(V), S(V) \# G)^{G} \cong \mathrm{HH}^{*}(S(V) \# G) .
$$

Proof. We constructed a specific choice of chain map $\Psi_{B}$ in Definition 4-1 above from the bar to the Koszul resolution of $S(V)$. Since $\Psi$ and $\Psi_{B}$ are homotopic by the Comparison Theorem, $\Psi_{g}^{*}=\Psi_{g, B}^{*}$ as maps on cohomology $\mathrm{HH}^{*}(S(V), S(V) \bar{g})$. But $\Psi_{g, B}^{*}=\Upsilon_{g, B}$ for any choice of $g$ and $B$ by Theorem 5-1, and hence $\Psi^{*}=\Upsilon$. By Proposition 2-8, these maps preserve $G$-invariant subspaces, and so $\Psi^{*}=\Upsilon$ on $\mathrm{HH}^{\circ}(S(V) \# G)$ as well.

Corollary 5-3. Let $g \in G$. On the cohomology $\mathrm{HH}^{*}(S(V), S(V) \bar{g})$, the map $\Upsilon_{g}=$ $\Upsilon_{g, B}$ is independent of choice of basis $B$ of eigenvectors of $g$ used in its definition. Hence, as a map on the cohomologies $\mathrm{HH}^{\circ}(S(V), S(V) \# G)$ and $\mathrm{HH}^{\circ}(S(V) \# G)$, $\Upsilon$ is independent of the choices of bases used in its definition.

Proof. By Corollary 5-2, $\Upsilon_{g}=\Psi_{g}^{*}$ on cohomology for any choice of chain map $\Psi$ from the bar complex to the Koszul complex of $S(V)$, independent of the choice of basis of eigenvectors of $g$ used to define $\Upsilon_{g}$. Hence, $\Upsilon$ is independent of choices of bases.

Corollary 5-4. The maps $\Upsilon$ and $\Phi^{*}$ are inverse isomorphisms on the cohomology $\mathrm{HH}^{*}(S(V), S(V) \# G)$ and on its $G$-invariant subalgebra $\mathrm{HH}^{*}(S(V), S(V) \# G)^{G} \cong$ $\mathrm{HH}^{*}(S(V) \# G)$. 
Proof. Again, in Corollary 5-2, we found that $\Upsilon=\Psi^{*}$ on cohomology for any chain map $\Psi$ from the bar to the Koszul complex. But any such $\Psi$ induces an automorphism on cohomology inverse to $\Phi^{*}$ by the Comparison Theorem.

This corollary actually follows from a stronger fact: $\Upsilon$ is a right-sided inverse to $\Phi^{*}$ on cochains, not merely on cohomology, for any choice of bases $\left\{B_{g}\right\}_{g \in G}$ defining $\Upsilon$. Indeed, a calculation shows directly that $\Phi^{*} \Upsilon=1$ on cochains $C^{*}$. We can see this fact yet another way. One can check that $\Psi_{B} \Phi=1$ on chains, and therefore $\Phi_{g}^{*}\left(\Psi_{B}\right)_{g}^{*}=1$ on cochains, for every $B$ and $g$. In Theorem 5-1, we saw that $\Upsilon_{g, B}=\left(\Psi_{B}\right)_{g}^{*}=\Psi_{g, B}^{*}$ as maps on cochains, for all $g$ in $G$ and for any basis $B$ of eigenvectors of $g$, and hence $\Phi^{*} \Upsilon=1$ as a map on cochains.

\section{Hochschild homology}

Our chain maps $\Psi_{B}$ of Definition 4-1 are useful in settings other than the cohomology of $S(V) \# G$. In this section, we obtain induced maps on Hochschild homology, and compare our induced maps on homology and cohomology with those in the literature. The Hochschild-Kostant-Rosenberg Theorem states that for smooth commutative algebras, Hochschild homology is isomorphic to the module of differential forms (i.e., the exterior algebra generated by the Kähler differentials); e.g., see [Weibel 1994, §9.4.2]. For noncommutative algebras, Hochschild homology provides a generalization of the notion of "differential forms". It is interesting to note that for some types of algebras (in particular for $S(V) \# G$ ), Hochschild homology and cohomology are dual (see [van den Bergh 1998] for the general theory and [Farinati 2005] for the case $S(V) \# G)$. In this section, we work over an arbitrary field initially, then over $\mathbb{C}$ in Theorem 6-4.

Let $M$ be any $S(V)^{e}$-module. Then $\Psi_{B}$ induces an isomorphism on Hochschild homology

$$
\mathrm{HH}_{.}(S(V), M):=\operatorname{Tor}_{\bullet}^{S(V)^{e}}(S(V), M)
$$

and on Hochschild cohomology

$$
\mathrm{HH}^{\circ}(S(V), M):=\operatorname{Ext}_{S(V)^{e}}(S(V), M)
$$

by applying the functors $M \otimes_{S(V)^{e}}-$ and $\operatorname{Hom}_{S(V)^{e}}(-, M)$, respectively, to the bar resolution (2-1) and to the Koszul resolution (2-4). This approach to obtaining maps on homology and cohomology has advantages over previous approaches in the literature which we explain now.

We obtain a map on Hochschild homology HH. $(S(V)):=\mathrm{HH}_{\bullet}(S(V), S(V))$, denoted by $\left(\Psi_{B}\right)_{*}$, by setting $M=S(V)$. At the chain level,

$$
\left(\Psi_{B}\right)_{*}: S(V) \otimes S(V)^{\otimes \bullet} \longrightarrow S(V) \otimes \wedge^{\bullet}(V) .
$$


A computation similar to that in the proof of Theorem 5-1 yields the following explicit formula for $\left(\Psi_{B}\right)_{*}$, valid over any ground field:

Theorem 6-1. Let $B=\left\{v_{1}, \ldots, v_{n}\right\}$ be a basis of $V$. Then as an automorphism on HH. $(S(V))$ at the chain level,

$$
\left(\Psi_{B}\right)_{*}\left(f_{0} \otimes f_{1} \otimes \cdots \otimes f_{p}\right)=\sum_{1 \leq i_{1}<\cdots<i_{p} \leq n} f_{0} \frac{\partial f_{1}}{\partial v_{i_{1}}} \cdots \frac{\partial f_{p}}{\partial v_{i_{p}}} \otimes v_{i_{1}} \wedge \cdots \wedge v_{i_{p}}
$$

for all $f_{0}, f_{1}, \ldots, f_{p} \in S(V)$.

Proof. Without loss of generality, assume that $f_{1}, \ldots, f_{p}$ are monomials, say

$$
f_{k}=\underline{v}^{\underline{\ell}^{k}}=v_{1}^{\ell_{1}^{k}} \cdots v_{n}^{\ell_{n}^{k}}
$$

for some $n$-tuple $\underline{\ell}^{k}=\left(\ell_{1}^{k}, \ldots, \ell_{n}^{k}\right)$. Then

$$
\begin{aligned}
& \left(\Psi_{B}\right)_{*}\left(f_{0} \otimes \underline{v}^{\underline{\ell}} \underline{1}^{1} \otimes \cdots \otimes \underline{v}^{\underline{\ell}}\right) \\
& =f_{0} \Psi_{B}\left(1 \otimes \underline{v}^{\underline{\ell}} \otimes \cdots \otimes \underline{v}^{\underline{\ell}} \underline{\underline{\ell}}^{p}\right) \\
& =f_{0}\left(\sum_{1 \leq i_{1}<\cdots<i_{p} \leq n} \sum_{0 \leq a_{i_{j}}<\ell_{i_{j}}^{j}} \underline{\underline{v}} \underline{\underline{Q}\left(\underline{\ell} ; i_{1}, \ldots, i_{p}\right)} \otimes \underline{v} \underline{\hat{Q}}^{\left(\ell ; i_{1}, \ldots, i_{p}\right)} \otimes v_{i_{1}} \wedge \cdots \wedge v_{i_{p}}\right) \\
& \left.=\sum_{1 \leq i_{1}<\cdots<i_{p} \leq n} \sum_{0 \leq a_{i_{j}}<\ell_{i_{j}^{j}}^{j}} \underline{\underline{v}} \underline{\underline{Q}} \underline{\ell} ; i_{1}, \ldots, i_{p}\right) f_{0} \underline{\underline{v}} \underline{\hat{Q}} \underline{\left(\ell ; i_{1}, \ldots, i_{p}\right)} \otimes v_{i_{1}} \wedge \cdots \wedge v_{i_{p}} \\
& =\sum_{1 \leq i_{1}<\cdots<i_{p} \leq n} \sum_{0 \leq a_{i_{j}}<\ell_{i_{j}}^{j}} f_{0} \underline{v} \underline{\underline{Q}} \underline{\left(\ell ; i_{1}, \ldots, i_{p}\right)} \underline{\underline{v}} \underline{\hat{Q}} \underline{\left(\ell ; i_{1}, \ldots, i_{p}\right)} \otimes v_{i_{1}} \wedge \cdots \wedge v_{i_{p}} \\
& =\sum_{1 \leq i_{1}<\cdots<i_{p} \leq n} \sum_{0 \leq a_{i_{j}}<\ell_{i_{j}}^{j}} f_{0} \underline{v}^{\underline{\ell}^{1}} \cdots \underline{v}^{\underline{\ell}^{p}} v_{i_{1}}^{-1} \cdots v_{i_{p}}^{-1} \otimes v_{i_{1}} \wedge \cdots \wedge v_{i_{p}} \\
& =\sum_{1 \leq i_{1}<\cdots<i_{p} \leq n} \ell_{i_{1}}^{1} \cdots \ell_{i_{p}}^{p} f_{0} \underline{v}^{\underline{\ell}} \cdots \underline{\underline{\ell}}^{\underline{\ell}} v_{i_{1}}^{-1} \cdots v_{i_{p}}^{-1} \otimes v_{i_{1}} \wedge \cdots \wedge v_{i_{p}} \text {, }
\end{aligned}
$$

where the product $\underline{v}^{\underline{\ell}} \cdots \underline{v}^{1} \underline{\ell}^{p} v_{i_{1}}^{-1} \cdots v_{i_{p}}^{-1}$ is computed in the ring of Laurent polynomials in $v_{1}, \ldots, v_{n}$. (Since $0 \leq a_{i_{j}}<\ell_{i_{j}}^{j}$, the result lies in $S(V)$ when the corresponding sum is nonempty.) The expression above is precisely that claimed in the theorem.

In case the ground field is $\mathbb{C}$ or $\mathbb{R}$, by the above theorem, our map $\left(\Psi_{B}\right)_{*}$ is precisely the map $J$ of [Halbout 2001]. Halbout gave an explicit homotopy $s$ showing that $J$ is a homotopy inverse to the canonical embedding of the de Rham complex into the Hochschild complex. In contrast, we see immediately that $\left(\Psi_{B}\right)_{*}$ induces an isomorphism on homology since $\Psi_{B}$ is itself a chain map.

For comparison, we give the map on Hochschild cohomology $\mathrm{HH}^{*}(S(V))$; this is simply the case $g=1$ of Definition 3-4, by Theorem 5-1: 
Theorem 6-3. Let $B=\left\{v_{1}, \ldots, v_{n}\right\}$ be a basis of $V$. Then as an automorphism on $\mathrm{HH}^{\circ}(S(V))$ at the chain level,

$$
\left(\Psi_{B}\right)^{*}(\alpha)\left(f_{1} \otimes \cdots \otimes f_{p}\right)=f_{0} \frac{\partial f_{1}}{\partial v_{j_{1}}} \cdots \frac{\partial f_{p}}{\partial v_{j_{p}}},
$$

when $\alpha=f_{0} \otimes v_{j_{1}}^{*} \wedge \cdots \wedge v_{j_{p}}^{*} \in \operatorname{Hom}_{\mathbb{C}}\left(S(V) \otimes \wedge^{p} V, S(V)\right), f_{0}, \ldots, f_{p} \in S(V)$.

Now we restrict our choice of field again to $\mathbb{C}$. Let $M=S(V) \# G$, and note that Hochschild homology decomposes just as does Hochschild cohomology:

$$
\mathrm{HH}_{\bullet}(S(V) \# G) \cong \mathrm{HH}_{\bullet}(S(V), S(V) \# G)^{G} \cong\left(\bigoplus_{g \in G} \mathrm{HH}_{\bullet}(S(V), S(V) \bar{g})\right)^{G}
$$

(see [Farinati 2005; Ştefan 1995]). Thus one is interested in the components HH. $(S(V), S(V) \bar{g})=\operatorname{Tor}_{\bullet}^{S(V)^{e}}(S(V), S(V) \bar{g})$, for each $g$ in $G$. A calculation similar to that in the proof of Theorem 5-1 yields the explicit formula in the next theorem for the induced map

$$
\left(\Psi_{B}\right)_{*}: S(V) \bar{g} \otimes S(V)^{\otimes \cdot} \rightarrow S(V) \bar{g} \otimes \bigwedge^{\bullet}(V) .
$$

Note that quantum differential operators surface (compare with Definition 3-4 of $\Upsilon$, which is equal to $\Psi_{B}^{*}$ by Theorem 5-1). We have not found these maps in the literature on Hochschild homology.

For $g$ in $G$, let $B=\left\{v_{1}, \ldots, v_{n}\right\}$ be a basis of $V$ consisting of eigenvectors of $g$ with corresponding eigenvalues $\epsilon_{1}, \ldots, \epsilon_{n}$. Write $g=s_{1} \cdots s_{n}$ where ${ }^{s_{i}} v_{j}=v_{j}$ for $j \neq i$ and ${ }^{s_{i}} v_{i}=\epsilon_{i} v_{i}$. Recall the quantum partial differential operators $\partial_{i}:=\partial_{v_{i}, \epsilon_{i}}$ of Definition (3-1).

Theorem 6-4. Let $g \in G$ and let $B=\left\{v_{1}, \ldots, v_{n}\right\}$ be a basis of $V$ consisting of eigenvectors for $g$. Then as an automorphism on $\mathrm{HH}_{.}(S(V), S(V) \bar{g})$ at the chain level,

$$
\left(\Psi_{B}\right)_{*}\left(f_{0} \bar{g} \otimes f_{1} \otimes \cdots \otimes f_{p}\right)=\sum_{1 \leq i_{1}<\cdots<i_{p} \leq n} f_{0}\left(\prod_{k=1}^{p} s_{1} s_{2} \cdots s_{i_{k-1}}\left(\partial_{i_{k}} f_{k}\right)\right) \bar{g} \otimes v_{i_{1}} \wedge \cdots \wedge v_{i_{p}}
$$

for all $f_{0}, f_{1}, \ldots, f_{p} \in S(V)$.

We make a few final comments about the appearance of our chain maps $\Psi_{B}$ in Hochschild cohomology. Again let $M=S(V) \# G$, and consider the map $\left(\Psi_{B}\right)^{*}$ on the Hochschild cohomology $\mathrm{HH}^{*}(S(V), S(V) \# G)$ for any basis $B$ of $V$. We observed (as a consequence of Theorem 5-1 and Definition 3-4) that $\left(\Psi_{B}\right)^{*}$ is given by quantum partial differential operators. The reader should compare with maps given in [Halbout and Tang 2010]: these authors define functions directly on cochain 
complexes (without first defining chain maps on resolutions) and then must prove that these functions are cochain maps. Again, our approach presents an advantage: We instead define one primitive chain map $\Psi_{B}$ from which induced cochain maps effortlessly spring. For example, $\left(\Psi_{B}\right)^{*}=\Upsilon$ is automatically a cochain map since $\Psi_{B}$ is a chain map by Theorem 4-4. The reader is cautioned that Halbout and Tang [2010] work only over $\mathbb{R}$, in which case Hochschild cohomology has a specialized description ( $V$ and $V^{*}$ are $G$-isomorphic in that setting, simplifying some aspects of homology and cohomology).

\section{Appendix: Proof of Theorem 4-4}

Let $V$ be a finite-dimensional vector space over any field. Fix a basis

$$
B=\left\{v_{1}, \ldots, v_{n}\right\}
$$

of $V$. Recall Definition 4-1 of the linear map $\Psi=\Psi_{B}$ from the bar resolution (2-1) to the Koszul resolution (2-4) of $S(V)$. We prove that $\Psi$ is a chain map, that is, $\Psi_{p-1} \delta_{p}=d_{p} \Psi_{p}$ for all $p \geq 1$.

A straightforward but tedious calculation shows that $\Psi_{0} \delta_{1}=d_{1} \Psi_{1}$, and we assume from now on that $p \geq 2$.

We first compute

$$
d_{p} \Psi_{p}\left(1 \otimes \underline{\underline{v}}^{\underline{\ell}} \otimes \cdots \otimes \underline{\underline{\ell}}^{\underline{\ell}} \otimes 1\right) .
$$

For each $j \in \mathbb{N}$, let $\delta[j]: \mathbb{N} \rightarrow\{0,1\}$ be the Kronecker delta function defined by

$$
(\delta[j])_{i}=\delta[j](i)= \begin{cases}1 & \text { if } i=j \\ 0 & \text { if } i \neq j .\end{cases}
$$

Then

$$
\begin{aligned}
& d_{p} \Psi_{p}\left(1 \otimes \underline{v}^{\underline{\ell}^{1}} \otimes \cdots \otimes \underline{v}^{\underline{\ell}^{p}} \otimes 1\right)
\end{aligned}
$$

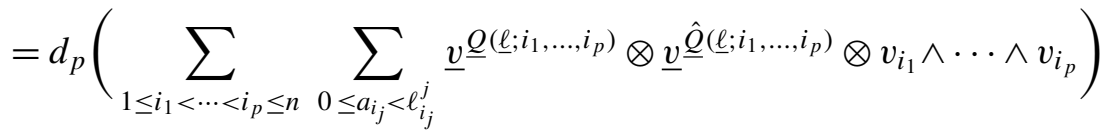

$$
\begin{aligned}
& \begin{array}{r}
\sum_{1 \leq i_{1}<\cdots<i_{p} \leq n} \sum_{0 \leq a_{i_{j}<\ell_{i_{j}}^{j}}} \sum_{m=1}^{p}(-1)^{m+1}\left(\underline{v} \underline{\underline{Q}}+\delta\left[i_{m}\right] \otimes \underline{v} \underline{\hat{Q}} \otimes v_{i_{1}} \wedge \cdots \wedge \hat{v}_{i_{m}} \wedge \cdots \wedge v_{i_{p}}\right. \\
\left.-\underline{v} \underline{\underline{Q}} \otimes \underline{\underline{v}} \underline{\underline{Q}}^{+\delta\left[i_{m}\right]} \otimes v_{i_{1}} \wedge \cdots \wedge \hat{v}_{i_{m}} \wedge \cdots \wedge v_{i_{p}}\right),
\end{array}
\end{aligned}
$$

where $\underline{Q}=\underline{Q}\left(\underline{\ell} ; i_{1}, \ldots, i_{p}\right)$ and $\underline{\hat{Q}}=\underline{\hat{Q}}\left(\underline{\ell} ; i_{1}, \ldots, i_{p}\right)$ are determined by the $a_{i_{j}}$ as in Definition 4-1. Now fix $m$ in the above expression. The factors $\underline{v} \underline{Q}+\delta\left[i_{m}\right]$ and $\underline{v} \underline{Q}$ differ only in the power of $v_{i_{m}}$. In the sum, the power $a_{i_{m}}$ ranges over the set $\left\{0, \ldots, \ell_{i_{m}}^{m}-1\right\}$, and thus the corresponding terms cancel except for the first term 
when $a_{i_{m}}=\ell_{i_{m}}^{m}-1$ and the second term when $a_{i_{m}}=0$. After all such cancellations, for each $m=1, \ldots, p$, what remains is

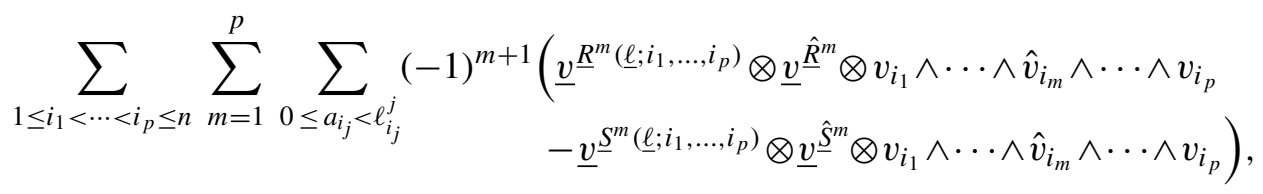

where the rightmost sum is over $a_{i}, \ldots, \hat{a}_{i_{m}}, \ldots, a_{i_{p}}$ and (abusing notation, as $\underline{R}, \underline{S}$ depend on fewer $a_{i j}$ 's than $\underline{Q}$ )

$$
\begin{aligned}
& \underline{R}^{m}\left(\underline{\ell} ; i_{1}, \ldots, i_{p}\right)_{i}= \begin{cases}\ell_{i_{m}}^{1}+\cdots+\ell_{i_{m}}^{m} & \text { if } i=i_{m}, \\
\underline{Q}\left(\underline{\ell} ; i_{1}, \ldots, i_{p}\right)_{i} & \text { if } i \neq i_{m},\end{cases} \\
& \underline{S}^{m}\left(\underline{\ell} ; i_{1}, \ldots, i_{p}\right)_{i}= \begin{cases}\ell_{i_{m}}^{1}+\cdots+\ell_{i_{m}}^{m-1} & \text { if } i=i_{m}, \\
\underline{Q}\left(\underline{\ell} ; i_{1}, \ldots, i_{p}\right)_{i} & \text { if } i \neq i_{m},\end{cases}
\end{aligned}
$$

and $\underline{\hat{R}}^{m}, \underline{\hat{S}}^{m}$ are defined by the equations

$$
\begin{aligned}
& \underline{v}^{\underline{R^{m}}} \underline{\ell}^{\left.\underline{\ell} ; i_{1}, \ldots, i_{p}\right)} \underline{v}^{\underline{\hat{R}}^{m}\left(\underline{\ell} ; i_{1}, \ldots, i_{p}\right)} v_{i_{1}} \cdots \hat{v}_{i_{m}} \cdots v_{i_{p}}=\underline{v}^{\underline{\ell}} \cdots \underline{\underline{\ell}}^{\underline{\underline{\ell}}}{ }^{p},
\end{aligned}
$$

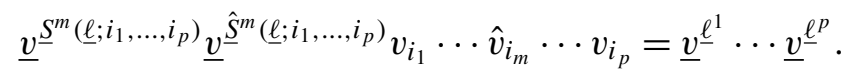

Consider the leftmost sum over $1 \leq i_{1}<\cdots<i_{p} \leq n$. If we replace a given $i_{m}$ in $\underline{S}^{m}$ by $i_{m}+1$ in $\underline{R}^{m}$ (provided $i_{m}+1<i_{m+1}$ ), keeping the others fixed, then

$$
\underline{S}^{m}\left(\underline{\ell} ; i_{1}, \ldots, i_{p}\right)=\underline{R}^{m}\left(\underline{\ell} ; i_{1}, \ldots, i_{m-1}, i_{m}+1, i_{m+1}, \ldots, i_{p}\right) .
$$

We thus have further cancellation, with the remaining terms coming from the first summand when $i_{m}=i_{m-1}+1$ and the second summand when $i_{m}=i_{m+1}-1$ :

$$
\begin{aligned}
& \sum_{m=1}^{p} \sum_{1 \leq i_{1}<\cdots<\hat{\imath}_{m}<\cdots<i_{p} \leq n} \sum_{0 \leq a_{i_{j}}<\ell_{i_{j}}^{j}}(-1)^{m+1} \\
& \left(\underline{v}^{\underline{\underline{R}}} \underline{\underline{R}}^{\left.\underline{\ell} ; i_{1}, \ldots, i_{m-1}, i_{m-1}+1, i_{m+1}, \ldots, i_{p}\right)} \otimes \underline{v}^{\hat{\underline{R}}^{m}} \otimes v_{i_{1}} \wedge \cdots \wedge \hat{v}_{i_{m}} \wedge \cdots \wedge v_{i_{p}}\right. \\
& \left.-\underline{v}^{\underline{S}}\left(\underline{\ell} ; i_{1}, \ldots, i_{m-1}, i_{m+1}-1, i_{m+1}, \ldots, i_{p}\right) \otimes \underline{v}^{\hat{S}^{m}} \otimes v_{i_{1}} \wedge \cdots \wedge \hat{v}_{i_{m}} \wedge \cdots \wedge v_{i_{p}}\right),
\end{aligned}
$$

where the rightmost sum is over all such $a_{i_{1}}, \ldots, \hat{a}_{i_{m}}, \ldots, a_{i_{p}}$.

Now consider the middle sum above ranging over all $1 \leq i_{1}<\cdots<\hat{\imath}_{m}<\cdots<$ $i_{p} \leq n$. If $m=1$, this sum does not include $i_{2}=1$, due to the left out entry $\hat{i}_{m}$. Similarly, if $m=p$, this sum does not include $i_{p-1}=n$. For the sake of later comparison, we add and subtract terms in the $m=1$ summand, corresponding to $i_{2}=1$, and in the $m=p$ summand, corresponding to $i_{p-1}=n$. These added and subtracted terms may be written with either notation, $\underline{R}$ or $\underline{S}$, so that the result 
looks the same as above except that now we include summands corresponding to $m=1, i_{2}=1$ and to $m=p, i_{p-1}=n$.

We next combine some of the terms. Consider the terms arising from a pair of subsequent indices $m$ and $m+1$ in the leftmost sum. We pair each summand of type $\underline{S}^{m}$ with a summand of type $\underline{R}^{m+1}$. Fix an integer $i$ and collect those summands (in the $m$-th sum) with $\underline{S}^{m}$-exponent for which $i_{m+1}=i$ and those summands (in the $(m+1)$-st sum) with $\underline{R}^{m+1}$-exponent for which $i_{m}=i$. Note that all these summands share the same sign. We compare the exponents

$$
\underline{S}^{m}\left(\underline{\ell} ; i_{1}, \ldots, i_{m-1}, i_{m+1}-1, i_{m+1}, \ldots, i_{p}\right)
$$

and

$$
\underline{R}^{m+1}\left(\underline{\ell} ; i_{1}, \ldots, i_{m}, i_{m}+1, i_{m+2}, \ldots, i_{p}\right)
$$

when $i_{m}=i=i_{m+1}$ and see that the power of $v_{i}$ ranges from $\ell_{i}^{1}+\cdots+\ell_{i}^{m-1}$ to $\ell_{i}^{1}+\cdots+\ell_{i}^{m-1}+\ell_{i}^{m}-1$ and then again from $\ell_{i}^{1}+\cdots+\ell_{i}^{m-1}+\ell_{i}^{m}$ to $\ell_{i}^{1}+\cdots+$ $\ell_{i}^{m}+\ell_{i}^{m+1}-1$ in this collection. Hence, we can simply rewrite the partial sum over this collection using the exponent

$$
\underline{Q}\left(\underline{\ell}^{1}, \ldots, \underline{\ell}^{m-1}, \underline{\ell}^{m}+\underline{\ell}^{m+1}, \underline{\ell}^{m+2}, \ldots, \underline{\ell}^{p} ; i_{1}, \ldots, \hat{\imath}_{m+1}, \ldots, i_{p}\right)
$$

instead. We obtain the following, in which the $m=1$ (unmatched $\underline{R}^{1}$ ) and $m=p$ (unmatched $\underline{S}^{p}$ ) sums have been singled out:

$$
\begin{aligned}
& \left.\sum_{1 \leq i_{2}<\cdots<i_{p} \leq n} \sum_{\substack{0 \leq a_{i_{j}<\ell_{i_{j}}^{j}} \\
(\text { for } j \in\{2, \ldots, p\})}} \underline{v} \underline{\underline{Q}} \underline{\underline{\ell}}^{2}, \cdots, \underline{\ell}^{p} ; i_{2}, \cdots, i_{p}\right)+\underline{\ell}^{1} \otimes \underline{v} \underline{\hat{Q}} \otimes v_{i_{2}} \wedge \cdots \wedge v_{i_{p}} \\
& +\sum_{m=1}^{p-1}(-1)^{m} \sum_{1 \leq i_{1}<\cdots<\hat{l}_{m}<\cdots<i_{p} \leq n} \sum_{0 \leq a_{i_{j}}<\ell_{i_{j}}^{j}}(\text { for } j \in\{1, \ldots, m-1\}) \\
& 0 \leq a_{i_{m+1}} \leq \ell_{i_{m+1}}^{m}+\ell_{i_{m+1}^{m+1}}^{m+1}-1 \\
& \left.0 \leq a_{i_{j}}<\ell_{i_{j}}^{j+1} \text { (for } j \in\{m+2, \ldots, p\}\right) \\
& \left.\underline{v} \underline{\underline{Q}} \underline{\ell}^{1}, \ldots, \underline{\ell}^{m-1}, \underline{\ell}^{m}+\underline{\ell}^{m+1}, \underline{\ell}^{m+2}, \ldots, \underline{\ell}^{p} ; i_{1}, \ldots, \hat{l}_{m}, \ldots, i_{p}\right) \otimes \underline{v} \underline{\hat{Q}} \otimes v_{i_{1}} \wedge \cdots \wedge \hat{v}_{m} \wedge \cdots \wedge v_{i_{p}}
\end{aligned}
$$

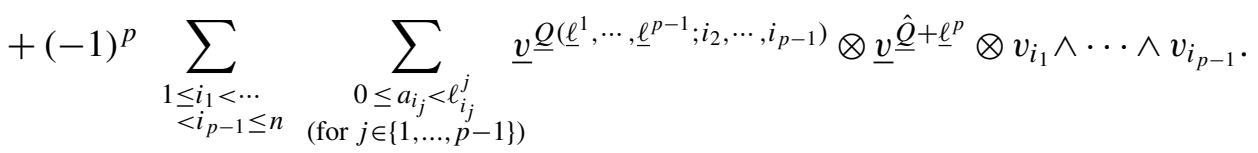

Now relabel indices so that each sum is taken over $1 \leq i_{1}<\cdots<i_{p-1} \leq n$. We 
obtain

$$
\begin{aligned}
& \left.\sum_{1 \leq i_{1}<\cdots<i_{p-1} \leq n} \sum_{0 \leq a_{i_{j}}<\ell_{i_{j}}^{j}} \underline{v} \underline{Q} \underline{\ell}^{2}, \cdots, \underline{\ell}^{p} ; i_{1}, \cdots, i_{p-1}\right)+\underline{\ell}^{1} \otimes \underline{v} \underline{\hat{Q}} \otimes v_{i_{1}} \wedge \cdots \wedge v_{i_{p-1}} \\
& +\sum_{m=1}^{p-1}(-1)^{m} \sum_{1 \leq i_{1}<\cdots<i_{p-1} \leq n} \sum_{0 \leq a_{i_{j}}<\ell_{i_{j}}^{j}}(\text { for } j \in\{1, \ldots, m-1\}) \\
& 0 \leq a_{i_{m}} \leq \ell_{i_{m}}^{m}+\ell_{i_{m}}^{m+1}-1 \\
& \left.0 \leq a_{i_{j}}<\ell_{i_{j}}^{j+1} \text { (for } j \in\{m+1, \ldots, p\}\right) \\
& \underline{v} \underline{Q}\left(\underline{\ell}^{1}, \ldots, \underline{\ell}^{m-1}, \underline{\ell}^{m}+\underline{\ell}^{m+1}, \underline{\ell}^{m+2}, \ldots, \underline{\ell}^{p} ; i_{1}, \ldots, i_{p-1}\right) \otimes \underline{v} \underline{\hat{Q}} \otimes v_{i_{1}} \wedge \cdots \wedge v_{i_{p-1}} \\
& +(-1)^{p} \sum_{1 \leq i_{1}<\cdots<i_{p-1} \leq n} \sum_{0 \leq a_{i_{j}<\ell_{i_{j}}^{j}}} \underline{v} \underline{Q}^{\left(\underline{\ell}^{1}, \cdots, \underline{\ell}^{p-1} ; i_{2}, \cdots, i_{p-1}\right)} \otimes \underline{v} \underline{\hat{Q}}+\underline{\ell}^{p} \otimes v_{i_{1}} \wedge \cdots \wedge v_{i_{p-1}} \\
& =\Psi_{p-1}\left(\delta_{p}\left(1 \otimes \underline{v}^{\underline{\ell}^{1}} \otimes \cdots \otimes \underline{v}^{\underline{\ell}^{p}} \otimes 1\right)\right) .
\end{aligned}
$$

This finishes the proof of Theorem 4-4.

\section{Acknowledgements}

The first author thanks Mohamed Barakat for useful conversations.

\section{References}

[van den Bergh 1998] M. van den Bergh, "A relation between Hochschild homology and cohomology for Gorenstein rings", Proc. Amer. Math. Soc. 126:5 (1998), 1345-1348. Erratum: 130:9 (2002), 2809-2810. MR 99m:16013 Zbl 0894.16005

[Braverman and Gaitsgory 1996] A. Braverman and D. Gaitsgory, "Poincaré-Birkhoff-Witt theorem for quadratic algebras of Koszul type”, J. Algebra 181:2 (1996), 315-328. MR 96m:16012 Zbl 0860.17002

[Căldăraru et al. 2004] A. Căldăraru, A. Giaquinto, and S. Witherspoon, "Algebraic deformations arising from orbifolds with discrete torsion", J. Pure Appl. Algebra 187:1-3 (2004), 51-70. MR 2005c:16013 Zbl 1055.16010

[Farinati 2005] M. Farinati, "Hochschild duality, localization, and smash products", J. Algebra 284:1 (2005), 415-434. MR 2005j:16009 Zbl 1066.16010

[Ginzburg and Kaledin 2004] V. Ginzburg and D. Kaledin, "Poisson deformations of symplectic quotient singularities", Adv. Math. 186:1 (2004), 1-57. MR 2005h:32072 Zbl 1062.53074

[Gordon and Smith 2004] I. Gordon and S. P. Smith, "Representations of symplectic reflection algebras and resolutions of deformations of symplectic quotient singularities", Math. Ann. 330:1 (2004), 185-200. MR 2006f:14013 Zbl 1062.14020

[Halbout 2001] G. Halbout, "Formule d'homotopie entre les complexes de Hochschild et de de Rham”, Compositio Math. 126:2 (2001), 123-145. MR 2002d:16010 Zbl 1007.16008

[Halbout and Tang 2010] G. Halbout and X. Tang, "Noncommutative Poisson structures on orbifolds”, Trans. Amer. Math. Soc. 362:5 (2010), 2249-2277. MR 2011b:58019 Zbl 05706807 
[Harris 1995] J. Harris, Algebraic geometry: a first course, Graduate Texts in Mathematics 133, Springer, New York, 1995. MR 97e:14001 Zbl 0779.14001

[Shepler and Witherspoon 2009] A. V. Shepler and S. Witherspoon, "Group actions on algebras and the graded Lie structure of Hochschild cohomology", preprint, 2009. arXiv 0911.0938v2

[Shepler and Witherspoon 2011] A. V. Shepler and S. Witherspoon, "Finite groups acting linearly: Hochschild cohomology and the cup product", Adv. Math. 226:4 (2011), 2884-2910. MR 2764878 Zbl 05854824

[Ştefan 1995] D. Ştefan, "Hochschild cohomology on Hopf Galois extensions", J. Pure Appl. Algebra 103:2 (1995), 221-233. MR 96h:16013 Zbl 0838.16008

[Weibel 1994] C. A. Weibel, An introduction to homological algebra, Cambridge Studies in Advanced Mathematics 38, Cambridge University Press, 1994. MR 95f:18001 Zbl 0797.18001

[Witherspoon 2006] S. Witherspoon, "Skew derivations and deformations of a family of group crossed products", Comm. Algebra 34:11 (2006), 4187-4206. MR 2007j:16016 Zbl 1135.16028

Communicated by Victor Reiner

Received 2010-03-17 Accepted 2010-06-11

ashepler@unt.edu

Mathematics Department, University of North Texas, 1155 Union Circle, Denton, TX 76203-1430, United States http://www.math.unt.edu/ ashepler/

sjw@math.tamu.edu

Department of Mathematics, Mailstop 3368, Texas A\&M University, College Station, TX 77843-3368, United States http://www.math.tamu.edu/ sarah.witherspoon/ 


\section{Algebra \& Number Theory}

www.jant.org

\section{EDITORS}

MANAGING EDITOR

Bjorn Poonen

Massachusetts Institute of Technology

Cambridge, USA

\author{
EDITORIAL BOARD CHAIR \\ David Eisenbud \\ University of California \\ Berkeley, USA
}

BOARD OF EDITORS

Georgia Benkart

Dave Benson

Richard E. Borcherds

John H. Coates

J-L. Colliot-Thélène

Brian D. Conrad

Hélène Esnault

Hubert Flenner

Edward Frenkel

Andrew Granville

Joseph Gubeladze

Ehud Hrushovski

Craig Huneke

Mikhail Kapranov

Yujiro Kawamata

János Kollár

Yuri Manin

Barry Mazur

Susan Montgomery
University of Wisconsin, Madison, USA

University of Aberdeen, Scotland

University of California, Berkeley, USA

University of Cambridge, UK

CNRS, Université Paris-Sud, France

University of Michigan, USA

Universität Duisburg-Essen, Germany

Ruhr-Universität, Germany

University of California, Berkeley, USA

Université de Montréal, Canada

San Francisco State University, USA

Hebrew University, Israel

University of Kansas, USA

Yale University, USA

University of Tokyo, Japan

Princeton University, USA

Northwestern University, USA

Harvard University, USA

University of Southern California, USA
Shigefumi Mori

Andrei Okounkov

Raman Parimala

Victor Reiner

Karl Rubin

Peter Sarnak

Michael Singer

Ronald Solomon

Vasudevan Srinivas

J. Toby Stafford

Bernd Sturmfels

Richard Taylor

Ravi Vakil

Michel van den Bergh

Marie-France Vignéras

Kei-Ichi Watanabe

Andrei Zelevinsky

Efim Zelmanov
RIMS, Kyoto University, Japan

Princeton University, USA

Emory University, USA

University of Minnesota, USA

University of California, Irvine, USA

Princeton University, USA

North Carolina State University, USA

Ohio State University, USA

Tata Inst. of Fund. Research, India

University of Michigan, USA

University of California, Berkeley, USA

Harvard University, USA

Stanford University, USA

Hasselt University, Belgium

Université Paris VII, France

Nihon University, Japan

Northeastern University, USA

University of California, San Diego, USA

\section{PRODUCTION}

contact@msp.org

Silvio Levy, Scientific Editor

Andrew Levy, Production Editor

See inside back cover or www.jant.org for submission instructions.

The subscription price for 2011 is US \$150/year for the electronic version, and \$210/year (+ \$35 shipping outside the US) for print and electronic. Subscriptions, requests for back issues from the last three years and changes of subscribers address should be sent to Mathematical Sciences Publishers, Department of Mathematics, University of California, Berkeley, CA 94720-3840, USA.

Algebra \& Number Theory (ISSN 1937-0652) at Mathematical Sciences Publishers, Department of Mathematics, University of California, Berkeley, CA 94720-3840 is published continuously online. Periodical rate postage paid at Berkeley, CA 94704, and additional mailing offices.

ANT peer review and production are managed by EditFLOw ${ }^{\mathrm{TM}}$ from Mathematical Sciences Publishers.

PUBLISHED BY

mathematical sciences publishers

http://msp.org/

A NON-PROFIT CORPORATION

Typeset in LATEX

Copyright $\odot 2011$ by Mathematical Sciences Publishers 


\section{Algebra \& Number Theory}

Volume $5 \quad$ No. $3 \quad 2011$

A categorical proof of the Parshin reciprocity laws on algebraic surfaces DENIS OSIPOV and XINWEN ZHU

Quantum differentiation and chain maps of bimodule complexes

ANNE V. SHEPLER and SARAH WITHERSPOON

Toric-friendly groups

MIKHAIL BOROVOI and ZINOVY REICHSTEIN

LuCHEZAR AVRAMOV, SRIKANTH B. IYENGAR and JOSEPH LIPMAN 